\title{
Daniel Alberto Panateri, El discurso del rey. El discurso jurídico alfonsí y sus implicancias políticas. Madrid: Dikynson, 2017, 289 pp.
}

Daniel Panateri, investigador del IMHICIHU-CONICET, presenta El discurso del rey: el discurso jurídico alfonsí y sus implicaciones políticas como el resultado en formato libro de su tesis doctoral, defendida en 2015. La obra ve la luz como publicación monográfica dentro de la línea de "Historia del Derecho" que mantiene el Instituto Figuerola de Historia y Ciencias Sociales de la Universidad Carlos III de Madrid. Tal y como advierte el autor, los resultados de esta investigación también dieron lugar a otras publicaciones en revistas científicas entre 2015 y 2017. En este sentido, parte del libro recoge y aúna lo contenido en dichas publicaciones.

La línea de trabajo de Panateri se centra en la legal history o historia del derecho, combinada con otras disciplinas afines, como la analítica del discurso, la crítica filológica o la ecdótica. El autor se dedica con ahínco a la crítica filológica de las fuentes conservadas de las Siete Partidas y al análisis de la obra y su discurso jurídicopolítico, demostrando un sólido dominio de las materias que va tratando. Gracias a un hábil manejo del contexto y de las herramientas conceptuales que presta cada disciplina abarcada, el carácter interdisciplinar de la investigación marca un salto cualitativo en cuanto a enfoque y método respecto a la línea marcada por otros trabajos sobre la obra jurídica de Alfonso X. Así, evita la tendencia a la interpretación reduccionista que mostraban los trabajos de la historia del derecho del siglo XX, así como la falta de contextualización de las investigaciones de carácter filológico. ${ }^{1}$

A nivel temático, el libro estudia la construcción del discurso regio en las Partidas de Alfonso X. Sobre el material trabajado (el texto de las Partidas) nota dos consideraciones que determinan el planteamiento de la investigación. Primero, la permanencia de la obra como objeto intelectual en la política española durante ocho siglos. Segundo, su constante crisis de identidad derivada de los intentos de estabilización del texto. Esto lleva a que no se estudie las Partidas como un código legal, sino como un discurso construido (o reelaborado, según la ocasión) constantemente desde el poder. Por ello, parte de un muestreo sincrónico centrado en dos momentos: la composición por Alfonso X en el siglo XIII y la edición y glosa de Gregorio López en 1555 para Carlos I. Analiza la evolución del discurso regio de uno a otro punto en la perspectiva diacrónica. De este modo, observa la transición semántica de los conceptos políticos en los procesos de relectura y apropiación en el tiempo. Estos conceptos políticos son principalmente dos, el de soberanía y el de ley.

En principio, se podrían haber tomado como puntos de estudio otras ediciones que mediaron entre las dos seleccionadas, como por ejemplo el Ordenamiento de Alcalá en 1348 o la edición de Montalvo en 1491. Ahora bien, se llama la atención sobre la

\footnotetext{
${ }^{1}$ A este respecto, el profesor Alfonso García Gallo destacó al poner en relación la evolución de la obra jurídica de Alfonso X con sus avatares político. Véase, como forma más acabada de sus planteamientos: "La obra legislativa de Alfonso X. Hechos e hipótesis", AHDE, 55 (1985): 495-704. De igual modo, acusó el estatismo ("Nuevas observaciones sobre la obra legislativa de Alfonso", AHDE, (1967): 609670) de Juan Antonio Arias Bonet en su edición de la Partida I (Alfonso X el Sabio. Primera Partida (Manuscrito Add. 20.787 del British Museum), ed. J.A. Arias Bonet (Valladolid: Universidad, 1975).
} 
importancia de los cambios operados por López debido a que comparten un contexto similar: la cuestión imperial, los enfrentamientos con los señores de la tierra y la puja regia por la soberanía.

El Libro se abre con una sólida introducción en la que Panateri expone su consideración sobre el conjunto de la obra jurídica alfonsí y sobre la tradición manuscrita de las Partidas. Aquí echa mano de la crítica filológica para enmarcar las posibilidades de estudio que ofrece el tema según el actual estado de las fuentes. Seguidamente, a nivel estructural, el cuerpo del trabajo se divide en tres partes o capítulos.

En el capítulo primero, "La tradición manuscrita y el fenómeno entrópico", se dirimen dos elementos preliminares del trabajo (p. 289). Primero, todo lo relacionado con la crítica filológica para justificar la selección de fuentes. En segundo lugar, se desarrolla el apartado teórico sobre el que se lleva a cabo el análisis.

En el primer capítulo, se analiza la naturaleza política de la glosa de Gregorio López desde su gestación, retraída hasta el siglo XIII. Procede así sobre un cotejo de manuscritos que busca identificar los materiales que este autor habría utilizado, no se trata de un cotejo del texto de López con la totalidad de manuscritos conservados de las Partidas, labor monumental que excedería con mucho y sin sentido alguno, el objetivo perseguido por Panateri. De este análisis se extraen dos cuestiones principales. Primero, la existencia de dos versiones diferentes del texto alfonsí, más o menos contemporáneas, denominadas versión legalista y versión sapiencial. Segundo, que la labor de cotejo y selección de López sobre la tradición manuscrita de Partidas se puede considerar como pura crítica filológica.

En cuanto a las cuestiones filológicas, se observa que, si bien la transmisión del texto no es lineal y su estado de conservación no permite establecer una tradición manuscrita precisa, considera posible que existiese un texto conocido como Partidas durante el reinado de Alfonso X, concretamente entre 1265 y 1272. A partir de aquí, notando la disparidad entre los manuscritos conservados, Panateri identifica las dos versiones antes referidas y añade una tercera de mediados del XIV, que mezcla elementos de ambas. El por qué de la existencia de varias versiones plantea una incógnita determinante para el desarrollo de la investigación.

Sobre ello toma claras posiciones en un debate acerca de la materialidad del texto original que, aunque ya es viejo, no se ha resuelto de todo. Nos referimos aquí a la problemática abierta por el profesor Alfonso García-Gallo en el año 1951 y mantenida hasta $1985^{2}$ sobre si Alfonso X habría llegado a conocer el texto terminado o Siete Partidas es producto de reelaboraciones posteriores a la muerte del rey Sabio. ${ }^{3}$ Panateri se suma al rechazo unánime (y lógico, hemos de añadir) a las tesis de García-Gallo y, en páginas posteriores dedicadas específicamente a la tradición manuscrita, afirma que las Partidas fueron "una variedad de textos de diferente carácter después de su propia

\footnotetext{
2 Alfonso García-Gallo, "El Libro de las leyes de Alfonso el Sabio", AHDE, XXI-XXII (1951-1952): 345-528. También, A. García-Gallo, "La obra legislativa".

${ }^{3}$ Véase José Luis Bermejo Cabrero, "García-Gallo ante la obra legislativa de Alfonso X", Cuadernos de Historia del Derecho, 18 (2011): 163-191.
} 
génesis en el siglo XIII" (p. 51). Lamentablemente, las limitaciones de tiempo y espacio no permiten entrar en la argumentación, a favor o en contra, sobre lo que llama la "teoría de la poligénesis" de Arias Bonet, ni de la propuesta de "redacciones sucesivas" de Craddock para plantear una hipótesis sobre la relación que mantendrían los manuscritos conservados. ${ }^{4}$

Al igual que se dedica tiempo y espacio a la crítica textual de las Partidas en la que podría haber sido su redacción original, se lo dedica al estudio de las fuentes sobre las que Gregorio López podría haber construido su edición glosada. Para ello, se transcribe el prólogo completo de dicha edición intentando localizar las que podrían haber sido sus lecturas base. Panateri determina que López tuvo a la vista varios manuscritos, entre ellos la edición de Montalvo, reimpresa hasta 1550 y el Ordenamiento de Alcalá de 1348. Toma y descarta conscientemente manuscritos conocidos por los especialistas del momento con el fin de estabilizar una determinada versión y el discurso que contiene. De este modo, establece una base sobre la que intervenir de forma directa con una glosa con la que construye su propia presencia dentro del texto alfonsí. Por ello considera que llevó a cabo una edición crítica, pues el texto no coincide por entero con ninguno de los conservados. Habría procedido sobre una elección de lecciones entre manuscritos de diferentes ramas de la tradición, aunque la filiación del texto final es claramente sapiencial.

Tendríamos, pues, el hecho contradictorio de que el glosador toma un texto sapiencial para generar una obra que, en sí misma, se piensa con carácter jurídico. Sobre esto, Panateri presenta dos hipótesis generales. Una, que las ediciones modernas son deudores de la estabilización del texto en tono sapiencial en el Ordenamiento de Alcalá de 1348. Otra, que el triunfo de la corriente sapiencial se produce dentro de un proceso más largo, en un contexto más amplio, que se remonta al siglo XII. Cabe puntualizar aquí que, al referirse al tema del viraje sapiencial de la prosa jurídica castellana, el autor se muestra siempre seguidor de Hugo Bizarri. ${ }^{5}$ A esto se suma, a continuación, una tercera posibilidad. Que el triunfo del sapiencialismo en las Partidas se deba al fracaso en el intento de consolidar la imagen del rey como legislador máximo. Así, la supervivencia del texto vendría condicionada por la pérdida de su carácter de imperativo jurídico.

A nivel epistemológico, Panateri toma un planteamiento que consideramos de gran interés por ser potencialmente aplicable. Entiende que el cambio operado en el discurso que contiene las Partidas a lo largo del tiempo debe ser estudiado desde un paradigma distinto al de continuidad/ruptura, que provocar dicotomías ficticias. Por ello recurre a un concepto propio de la teoría de la comunicación, como es el de entropía, en su aplicación al estudio histórico de Rodríguez Velasco. ${ }^{6}$

\footnotetext{
${ }^{4}$ En la página 51, Panateri refiere a: J. A. Arias Bonet, Alfonso X el Sabio, XLVII-CIII; J.R. Craddock, "La cronología de las obras legislativas de Alfonso X el Sabio", AHDE, 51 (1981): 365-418.

5 Hugo Bizzarri, "Las colecciones sapienciales castellanas en el proceso de reafirmación del poder monárquico (siglos XII y XIV)", Cahiers de linguistique hispanique médiévale, 20 (1995): 35-73.

6 Jesús Rodríguez Velasco, "La urgente presencia de Las Siete Partidas", La Corónica, vol. 38, 2 (2010): 97-134.
} 
En su uso personal, Panateri entiende la variación entrópica del discurso en las Partidas como un proceso que se repite debido a la gran maleabilidad política del texto. Este enfoque permite ver cómo la obra se va adaptando a cada nuevo contexto de crisis monárquica, perdiéndose su entidad original hasta el punto de que, como en el caso de la glosa de López, interesa más como contenedor ideológico que por su propio contenido. Hace notar, así, que Siete Partidas se convierte en un referente absoluto del orden regio, manifestado a través del monopolio legislativo sobre la totalidad de la materia social. De igual modo, señala un proceso metonímico de identificación entre el rey y la ley, entre la imago regis y la imago legis (pp. 49-50).

Con la identificación del rey y la ley mediante una relación metonímica, Panateri argumenta una de sus tesis centrales: que las Partidas asentaron el derecho como un elemento permanente sobre el que se sustentó del discurso del poder monárquico ibérico. Con esta afirmación entra en un diálogo con tres corrientes historiográficas distintas en el estudio de la construcción y legitimación del poder regio. Primero, la corriente centrada en el modelo sacramental franco y anglosajón, que en España fue desarrollada por Nieto Soria. Segundo, la sostenida por Ruiz y Linehan, que niegan la presencia de la sacralidad, pero no aportan un elemento sustitutivo. En tercer lugar, está la apostura sostenida por Rucquoi y Rodríguez Velasco sobre que el elemento central de la construcción del poder regio en la Península Ibérica fue el derecho. ${ }^{7}$ Será esta la que toma Panateri, apartándose así del concepto Kantorowiano de "teología política". 8

Los capítulos dos y tres analizan la construcción del discurso regio en las Partidas a través de dos dimensiones. Una, la interna, referente al enfrentamiento entre el rey y la nobleza. Aquí, el discurso se construye a través de la dialéctica entre la ley del rey y otras formas normativas de raíz consuetudinaria. La otra, la externa, que coloca las Partidas en el contexto internacional, en el conflicto entre el papado y el imperio medieval. En este caso, el discurso se articula sobre la definición y delimitación de dos esferas de poder separadas. En ambos casos, el análisis procede ordenadamente, aplicándose primero al caso de Alfonso X en el siglo XIII y, después, al de Gregorio López en el XVI.

En el capítulo segundo, "La ley frente al uso, la costumbre y el fuero. Construcción del espacio interno" (pp. 94-158), se estudia la construcción del discurso jurídico sobre la soberanía regia en la Castilla Medieval y la España Moderna. Es de

\footnotetext{
${ }^{7}$ José Manuel Nieto Soria., "El imperio medieval como poder público: problemas de aproximación a un mito político", XXIII Semana de Estudios Medievales de Estella: Poderes públicos en la Europa Medieval: Principados, Reinos y Corona (Pamplona: 1997); Id., "La coronación del rey: los símbolos y la naturaleza de su poder", en Miguel Rodríguez Llopis (coord.), Alfonso X y su época (Barcelona: Carroggio, 2001), 127-151; e Id., "Tiempos y lugares de realeza sagrada en la Castilla de los siglos XIIXV", Annexes des Cahiers de linguistique et de civilisation hispaniques médiévales, 15 (2003): 263-284. Teofilo F. Ruiz, "Une royauté sans sacré: la monarchie castillane du bas Moyen Âge", Annales, 3 (1984): 429-453. Peter Linehan, "Pseudo-historia y pseudo-liturgia en la obra alfonsina", en A. Pérez Martín (ed.), España y Europa, un pasado jurídico común (Murcia: Instituto de Derecho Común, 1986), 259274. Adeline Rucquoi, "De los reyes que no son taumaturgos: los fundamentos de la realeza en España", Temas Medievales, 5 (1995): 163-186; e Id., Rex, Sapientia, Nobilitas. Estudios sobre la Península Ibérica Medieval (Granada: Universidad de Granada, 2006). Jesús Rodríguez Velasco, Ciudadanía, soberanía monárquica y caballería. Poética del orden de caballería (Madrid: Akal, 2009).

${ }^{8}$ Ernst Kantorowicz, Los dos cuerpos del rey. Un estudio de teologíapolítica medieval (Madrid: Alianza, 1985).
} 
destacar que aquí, Panateri puntualiza cómo el concepto de "Señorío Natural" desarrollado por los juristas alfonsíes se aplica en dos direcciones. En vertical, implicando la relación de obediencia entre el rey y el pueblo y en horizontal cohesionando a toda la gente de una tierra bajo un mando único.

Para el estudio de este proceso de formación discursiva se centra en los dos primeros títulos de la Partida I, haciendo hincapié en las diferencias entre la primera y la segunda redacción del texto, antes y después de la revuelta nobiliaria de 1272. En la primera, contenida en el ms. Add. 20.787 del British Museum, se igualan la ley y el fuero, se omiten el uso y la costumbre y se otorga al rey el monopolio de las capacidades legislativas. Esta redacción sería el primer ejemplar que tenemos de la corriente legalista. A partir de 1272 se produciría el giro sapiencial del texto, dando cabida a las exigencias de la nobleza con la introducción del uso, la costumbre y el fuero como formas normativas consuetudinarias que chocan con el proyecto jurídico regio.

De igual modo, Panateri hace notar que este virage permitió profundizar en la teoría política que se despliega, desarrollando la identificación metonímica entre el rey y la ley. En su primera redacción, las Partidas son menos sensibles al concepto romano de legibus solutus, que implica la libertad del soberano con respecto a la ley, pero tampoco se contempla mención alguna a la costumbre. A este efecto, la explicación del autor es que existió una relación inversamente proporcional entre la capacidad efectiva de mando de Alfonso X y el fortalecimiento de su teoría política.

Por el otro lado, para el siglo XVI plantea una situación diferente. Panateri nota cómo la Corona ya no mantiene un conflicto de poder con los grandes señores del reino. Es por ello que en la glosa de Gregorio López no se profundiza en el tema de la normatividad consuetudinaria. Todo lo contrario, estas formas se presentan como totalmente sometidas juridizadas, sometidas a la ley y reducidas en su aplicación a la resolución de conflictos locales por mano de un juez.

En el tercer capítulo, "El rey frente al papa y el emperador: construcción del espacio externo", se estudia la relación entre el poder papal y el imperial, tal y como se expone en cada texto de Partidas trabajado.

El planteamiento jurídico alfonsí implica la existencia de dos jurisdicciones separadas en el panorama internacional, ambas instituidas por Cristo y que representan, respectivamente, su doble realidad de hombre y deidad. Aquí, el desarrollo del concepto de "Señor Natural", a nivel internacional, choca con las pretensiones papales, pues no reconoce otro domino que no pueda ser revocado en virtud de la diferencia cualitativa respecto al vínculo de fidelidad al emperador.

Alfonso X representa un poder regio autónomo frente al poder papal, a la vez que se limita la acción de los dirigentes eclesiásticos a la esfera estrictamente espiritual. En cuanto a la ascendencia de su poder, ambas figuras rey-emperador y papa se presentan como vicarios de Cristo, pero se distinguen por tener jurisdicciones diferenciadas. Así, papado e imperio, o poder eclesiástico y secular, quedan separados por las funciones que deben desempeñar. Mientras la Iglesia queda recluida a la enseñanza de la doctrina 
y el cumplimiento de los sacramentos, al poder laico le corresponder gobernar y legislar. En definitiva, las Partidas componen una estructura ideológica que apuesta por maximizar la esfera de aplicación del poder regio, favoreciendo a la esfera temporal frente a la espiritual.

Gracias a este tipo de posiciones, Alfonso X se labró una mala reputación para su persona y obra en la corte papal. Así, la rehabilitación de Partidas por Gregorio López comporta un problema ideológico para la corte de un Carlos I.

A la hora de confrontar los dos textos, lo primero que llama la atención es que, mientras Alfonso X mostraba una división bastante clara de las esferas imperial-laica y papal-eclesiástica, López reivindica la potestad papal absoluta. Se produce así una contradicción con respecto al planteamiento imperial alfonsí. En este sentido, podemos establecer dos intenciones de la glosa de López siguiendo la división que Panateri hace en la estructura del libro: política interna y externa. En el nivel interno, Panateri parte de la postura de Villacañas, ${ }^{9}$ considerando que Carlos I actúa casi de forma permanente desde los Países Bajos, centrado en un proyecto imperial paneuropeo, en el que el reino de España es una simple base territorial del poder. Así, la glosa de López se centraría en la voluntad de suplir el vacío físico del monarca con la identificación simbólica de este con el texto alfonsí. A nivel externo, se busca la confraternización con el papado para que este legitime y consolide la adscripción de las Indias Occidentales a la Corona de Castilla.

A lo largo de todo el libro, lo que más destaca del estudio de Daniel Panateri es el análisis que realiza sobre el concepto de soberanía regia en su proceso de formación dentro del discurso regio a lo largo de trescientos años, desde Alfonso X a Gregorio López. De este modo, nota cómo lo que en el siglo XIII plantea un conflicto de poderes por la confrontación de jurisdicciones, en el XVI es un principio plenamente asentado e indiscutido. De igual modo, es de alabar la precaución epistemológica del autor al estudiar el proceso evolutivo del discurso, evitando identificar elementos que permitan fijar o definir una idea de soberanía (como ya se ha dicho, inexistente en aquel momento); más bien el autor centra en "la relación creativa entre texto y contexto" ( $\mathrm{p}$. 229). Es aquí donde entraría en juego el carácter entrópico del texto, mostrando su alto valor simbólico como instrumento ideológico al servicio del poder regio.

José Ángel Salgado Loureiro

Universidad de Santiago de Compostela

asalgado.loureiro@gmail.com

Fecha de recepción: 12 de junio de 2018.

Fecha de aceptación: 14 de junio de 2018.

Publicación: 30 de junio de 2018.

\footnotetext{
${ }^{9}$ José Luis Villacañas, ¿Qué imperio? (Córdoba: Almuzara, 2008).
} 